\title{
Treatments for Post-traumatic Stress Disorder: Pharmaceutical and Electrophysiologic Considerations
}

Dewleen G. Baker, M.D..$^{1,2,5,{ }^{*}}$
Immanuel Lerman, M.D. ${ }^{1,2,3,6}$
Emmanuel P. Espejo, Ph.D..$^{1,5}$
Robert McLay, M.D., Ph.D. ${ }^{4,5,6}$

\author{
Address \\ ${ }^{1}$ VA San Diego Healthcare System, San Diego, CA, USA \\ ${ }^{2}$ VA Center of Excellence for Stress and Mental Health, (116A) 3350 La Jolla Village \\ Dr, San Diego, CA 92161, USA \\ ${ }^{3}$ Department of Anesthesia, University of California, San Diego, CA, USA \\ ${ }^{4} \mathrm{Naval}$ Medical Center San Diego, San Diego, CA, USA \\ ${ }^{*}, 5$ Department of Psychiatry, San Diego School of Medicine, University of Califor- \\ nia, 3350 La Jolla Village Drive 116B, San Diego, CA 92161, USA \\ Email: dgbaker@ucsd.edu \\ Email: ilerman@ucsd.edu \\ Email: eespejo@ucsd.edu \\ ${ }^{6}$ Perlman Medical Office, UCSD Center for Pain Medicine, 9350 Campus Point Drive, \\ Suite 2C, La Jolla, CA, USA \\ Email: rmclay1@yahoo.com \\ Published online: 17 February 2015 \\ (C) Springer International Publishing AG (outside the USA) 2015
}

This article is part of the Topical Collection on Post-Traumatic Stress Disorders

Keywords Post-traumatic stress disorder (PTSD) - Pharmacotherapy • Psychotherapy •

Vagal nerve stimulation (VNS) - Transcranial magnetic stimulation (TMS) - Neural network

\section{Opinion statement}

Most individuals who experience traumatic events remain asymptomatic or symptoms resolve quickly, but in a minority who develop post-traumatic stress disorder (PTSD), symptoms can persist for decades and can be associated with psychiatric and medical comorbidities and poor social and occupational functioning. Currently, first-line pharmacotherapies are selective serotonin reuptake inhibitors (SSRIs) and serotoninnorepinephrine reuptake inhibitors (SNRIs) for core symptoms and in individuals with unremitting nightmares, prazosin, is commonly used and often effective. This review appraises these commonly used evidence-based treatments and their conceptual underpinnings. It follows with a discussion of concepts underlying emerging treatment research and development, in particular the use of combined psychotherapeutic-pharmacologic 
approaches and electro-physiological technologies that stimulate neural circuits and their potential uses. While the evidence for combined psychotherapy and either pharmacological or physiological treatments is only now emerging, some of these modalities appear promising.

\section{Introduction}

Post-traumatic stress disorder (PTSD), as a diagnostic entity, emerged in the early 1980s when the diagnostic criteria were first included in the Diagnostic and Statistical Manual for Mental Disorders, Version III (DSM-III). These criteria have since been revised to the most recent published in DSM-5 [1]. PTSD is atypical among the mental health disorders in that the disease model for symptom emergence is similar to that for infectious diseases in which the precipitation of symptoms follows exposure of a vulnerable host to a pathogenic vector, i.e., an infectious organism in the case of infections and a traumatic event in the case of PTSD. The vector is incorporated into the DSM-5 diagnostic criteria as criterion A defined as exposure to actual or threatened death, serious injury, or sexual violation. The event is either experienced directly, by witnessing it in person as it occurred to others, by learning that it occurred to a family member or close friend, or by experiencing repeated or extreme exposure, such as in the case of first responders [1]. Where rates of exposure have been measured, the lifetime prevalence of the exposure to a traumatic event is high and cumulative over a lifetime, with putative rates ranging from 17 to $89 \%$ [2-5]. There is credible evidence that the risk for PTSD follows a dose-response relationship, increasing with duration and intensity of trauma exposure, and that some types of events are more 'pathogenic' than others, although there is no evidence that the biological mechanism of injury varies by trauma type $[6-8,9 \bullet]$.

The behavioral manifestations that result from exposure to a category A event constitute further the DSM-V diagnostic criteria for PTSD, all associated with the event, including the presence of intrusive symptoms (criteria B); persistent avoidance of stimuli, e.g., avoidance of thoughts/feelings and avoidance of external reminders (criteria C); negative alterations in cognitions/ mood, e.g., difficulty remembering, exaggerated negative beliefs/expectations, distorted cognitions, and anhedonia (criteria D); and marked alteration in arousal or reactivity, e.g., irritable behavior, angry outbursts, reckless or self-destructive behavior, hypervigilance, and physiological arousal (criteria E). To qualify for the diagnosis, PTSD symptoms must be present for at least 1 month (criteria F) and must cause either clinically significant distress or functional impairment (criteria G) [1]. The disorder can begin acutely and fail to resolve or emerge over time (from months to years later) [1]. While the common vocabulary provided by DSM diagnostic criteria for PTSD has formed a foundation for advancements in research, inherent in the behaviorally based definition are conceptual constraints that can impede biologically based disease discovery. We discuss current evidence-based PTSD treatment, followed by a discussion of concepts from the neuroscience of fear learning and memory, now actively being researched as they relate to PTSD treatment. These concepts which integrate the new biological (molecular, cellular, and neural connectivity) knowledge offer a translational platform for novel physiologic and pharmacologic approaches to PTSD treatment development.

\section{Pharmacotherapy: evidence base}

Pharmacotherapy is a common treatment for PTSD $[10 \bullet, 11]$. The pharmacy records of the US Department of Veterans Affairs in 2004 show that most veterans diagnosed with PTSD received psychotropic medication ( $80 \%)$; among those, $89 \%$ were prescribed antidepressants, $61 \%$ anxiolytics or sedative hypnotics, and $34 \%$ antipsychotics [10•]. Data from the Market scan database, a database that compiles claims from US private insurers, shows that medication use is common in adult civilians with PTSD, with $60 \%$ receiving 


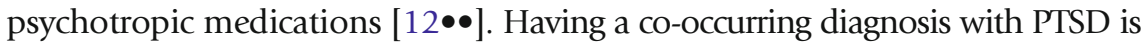
the most robust predictor of medication use for both the veterans and civilians, and whereas disease-specific use of medications for both PTSD and co-occurring disorders is common, much use is unrelated to the diagnosis, i.e., it appears to target specific symptoms (e.g., insomnia, anxiety, nightmares, and intrusive imagery), rather than the diagnosed illness as a whole $[10 \bullet, 11,12 \bullet \bullet, 13,14]$.

Overall, the clinical use of pharmaceutical treatments for PTSD has changed little in the past decade, and adequately powered and well-designed PTSD medication trials are relatively few $[12 \bullet \bullet, 15,16]$. In fact, the Institute of Medicine and the National Institute of England have argued that there is insufficient evidence to show proven efficacy of any drug or drug class, although the strongest evidence (i.e., largest number of subjects studied) has been with selective serotonin reuptake inhibitors (SSRIs), considered the treatment firstline pharmaceutical treatment for PTSD and the only class of drugs approved by

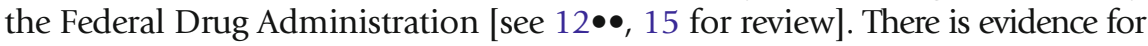
the efficacy of serotonin-norepinephrine reuptake inhibitors (SNRIs) for PTSD as well, but the evidence base is smaller than that for SSRIs, and SNRIs are not FDA approved [17, 18].

An example of a medication being used to target specific PTSD symptoms is prazosin, an $\alpha 1$-adrenergic blocker that has been increasingly accepted as a treatment for PTSD-related nightmares. Its use is supported by an accumulating number of clinical trials, most of them positive $[14,19 \bullet]$. Until the past few years, the approach to pharmaceutical development for PTSD has relied on goals of either mitigation of global PTSD symptoms, predicated on normalizing putative underlying pathological processes, or upon mitigation of targeted symptoms, e.g., nightmares, by modifying biological processes driving those

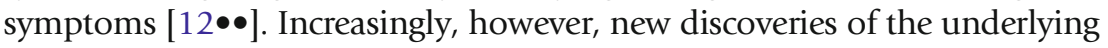
genetics, gene expression, molecular biology, and cell signaling of neurotransmitter systems responsive to stress offer tantalizing new pharmaceutical targets. However, since the specific mechanism(s) of injury in PTSD are unknown, the choice of which targets will effectively reduce overall symptoms has remained elusive, and few large-scale trials have been implemented, although some compounds, e.g., nepicastat (dopamine beta-hydroxylase antagonist), ganaxolone (synthetic analogs of allopregnenolone, a neurosteriod), mifepristone (glucocorticoid antagonist), and neuropeptide-Y, are undergoing small clinical trials in humans $[20,21]$.

In testing of genetic overlap for PTSD with bipolar disorder, major depressive disorder (MDD), and schizophrenia (SCZ) cohorts from the Psychiatric Genomics Consortium, PTSD diagnosis was observed to be associated with risk of bipolar disorder, but not MDD or SCZ; however, a recent study using the Positive and Negative Syndrome Scale (PANSS) showed some overlap in symptom structure between PTSD and SCZ, except that PTSD patients were less symptomatic on psychosis-related factors and more symptomatic on depression [22, 23]. Despite symptom structure similarities, however, a large, randomized double-blind placebo-controlled multicenter trial that tested the efficacy of 6-month treatment with risperidone in veterans with SSRI-resistant PTSD failed to show a significant reduction in PTSD symptoms when compared with placebo [24].

While some treatment trials targeting general PTSD symptoms are ongoing, recent pharmaceutical development has focused on strategies to modulate transmitter systems involved in learning and memory, with the key 
conceptual underpinnings of this research being to elucidate and modify the neurobiology of fear acquisition, extinction, and memory reconsolidation [25]. Likewise, neural networks involved in fear memory acquisition and maintenance are key targets of inquiry for the development of physiological interventions [26-28]. This targeted approach holds promise but may have limits in its ability to address the full range of post-trauma pathology $[29 \bullet \bullet]$.

\section{Underlying concepts and biology}

Memory acquisition and consolidation in organisms as complex as humans involve multiple layers of neural activity from the macro- to micro-level, i.e., 1) system-level communication (neural networks), 2) cell-to-cell communication (neurotransmitter systems), and 3) intracellular changes (epigenetic and protein) [30-32]. The core pathology of PTSD, fear memories, are long lasting and intensely emotion laden, but recent research shows memory longevity and strength to be a dynamic process that is dependent on contextual and biological influences [33, 34]. At the core of fear memory research design are concepts of fear extinction and reconsolidation, which are also the conceptual basis for cognitive-based verbal treatments for PTSD.

Most simply, extinction learning can be defined as learning in which conditioned responding to a stimulus decreases when the reinforcer is omitted. Extinction learning is thought to be new learning that overrides prior aversive memory. Thus, in this paradigm, extinction does not alter the threat memory itself but, instead, regulates its expression through an inhibitory (cognitive) influence of the pre-frontal cortex (PFC) over the amygdala (emotions). Like other forms of learning, extinction occurs in three phases: acquisition, consolidation, and retrieval, each of which depends on specific structures (amygdala, pre-frontal cortex, and hippocampus in particular) and molecular mechanisms (receptors and signaling pathways) $[35,36]$. A number of pharmacological reviews have been published on novel compounds that facilitate consolidation and retrieval of extinction; some of these compounds are under study for use in PTSD treatment, and others are being proposed based on recent pre-clinical research $[12 \bullet \bullet, 20,37 \bullet \bullet]$.

Whereas extinction is thought to create a new, competing memory trace that decreases fear expression, reconsolidation is hypothesized to be process involving protein synthesis by which memories, when reactivated, enter a transient, labile state that is followed by a re-stabilization, labeled reconsolidation. Reconsolidation mediates memory maintenance and updating, including strengthening or diminishment; memory diminishment can be accomplished by use of a protein inhibitor, e.g., anisomycin, but most protein inhibitors are too toxic for human use. Consequently, pre-clinical research on memory reconsolidation is proceeding rapidly, but most human reconsolidation research have been limited to propranolol, which interferes with the noradrenergic signaling thought to be necessary to strengthen and encode the emotional components of memory $[38,39]$. During the reconsolidation process, memory persistence can be influenced by various means (behavioral, physiological, and pharmaceutical) that lead to the potential memory updating (reviewed in detail by Agren) [40••]. 


\section{Cognitive behavioral approaches: state of the art}

Two cognitive behavioral (CBT) treatments, prolonged exposure (PE) and cognitive processing therapy (CPT), have a strong evidence base [15, 41, 42]. Typically, PE is conducted in 8 to 15 sessions, lasting 60 to 90 min each and involves imaginal exposure in an office setting as well as in vivo exposure, i.e., systematically approaching safe situations that are perceived to be dangerous or are avoided because they are reminders of the trauma. Treatment procedures (i.e., exposure to trauma-related cues) are posited to assist in the development of new associations that compete with (or inhibit) pathological fear memories in PTSD, i.e., extinction learning. CPT is based on a social cognitive theory of PTSD which holds that maladaptive construal of the trauma leads to pathological emotional responses and behaviors, and that changing maladaptive beliefs associated with the trauma are posited to result in recovery. While the treatment protocol in its original form includes a specific exposure component involving writing a detailed narrative of the trauma and reading it aloud in session, a version of the protocol omitting exposure to the trauma narrative has also been developed and has been demonstrated to be equally effective [43]. The underlying biological mechanisms, extinction, and reconsolidation remain under investigation at the pre-clinical and clinical levels as they apply to these treatment modalities [36, 44-46]. While there is solid evidence for effectiveness of these CBT treatments for PTSD, they are not universally beneficial. In fact, non-response to CBT in individuals with PTSD can be as high as $50 \%$, leaving considerable room for improvement from new approaches, such as augmen-

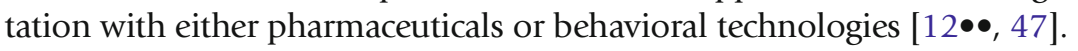

\section{Pharmacologic and combined pharmacologic/behavioral approaches}

Any salutatory effect of pharmaceutical augmentation with CBT could be hypothesized to be simply additive, conceived of as a facilitator of extinction learning, reconsolidation, or both. Sertraline has effects on fear conditioning and on pre-frontal-amygdala connectivity $[48,49]$. In a series of studies, SSRIs (sertraline and paroxetine) were add-ons to CBT studies, or visa versa, with mixed results [see [50, 51 - for review]. In a small study, in which subjects were randomized to SSRI (paroxetine) with CBT augmentation or CBT alone, the paroxetine augmentation initially appeared to be more effective, but the advantage disappeared by follow-up [50]. The authors speculated that the initial enhanced efficacy of the combined treatment reflected additive mechanisms but based on serotonin effects on fear learning, other mechanisms may be at play [48-50].

In contrast to use of adjunct SSRI-CBT treatment, recently studied CBT augmentation is predicated upon the enhancement of fear extinction by the pharmaceutical agent; thus, the drug is given intermittently, proceeding psychotherapy, rather than continuously as has been the case with SSRI augmentation. While there are many putative fear extinction enhancers identified in pre-clinical studies, few have yet been studied in humans, the first being $d$ - 
cycloserine (DCS), an $N$-methyl- $d$-aspartate (NMDA) glutamate receptor partial agonist [52]. The treatment trials have recently been reviewed; results are mixed $[51 \bullet, 53]$. In the study by Litz et al., DCS augmentation, combined with imaginal exposure (six sessions), did significantly worse than placebo [54]. Likewise, De Kleine et al. found no overall effect of DCS on augmentation of PE (12 sessions), but did report higher symptom reduction between therapy sessions in a subgroup with severe PTSD symptoms [55]. Both of these studies used $50 \mathrm{mg}$ DCS an hour prior to the exposure treatments. The other two trials that used virtual reality exposure (VRE) in conjunction with DCS were more positive. In a randomized, double-blind, placebo-controlled trial in which the subjects were given $100 \mathrm{mg}$ of DCS or placebo, Difede et al. showed earlier and greater improvement in the VRE-DCS group than the VRE-placebo group, as well as significantly greater (46 vs $8 \%$ at post-treatment; 69 vs $17 \%$ at 6 months) remission rates [56]. The study by Rothbaum et al. of 167 subjects was not as positive. A primary analysis of the outcome showed no advantage of DCS augmentation, but based on a secondary analysis, alprazolam augmentation impaired recovery whereas DCS enhanced virtual reality outcome in patients who demonstrated within-session learning [57].

These findings highlight the complexity of memory modulation, suggesting that similar to findings observed in animal models, extinction or enhancement of fear memories depends on contextual and biological factors that may vary

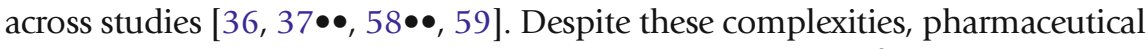
extinction learning enhancers are an extremely active area of pre-clinical inquiry, with many putative new pharmaceutical enhancers being proposed for future clinical trials in humans, especially those targeting glutamate signaling, but completed human trials are few [20]. One trial of rapamycin, an inhibitor of mTOR kinase (a critical regulator of mRNA translation known to be involved in various long-lasting forms of synaptic and behavioral plasticity), is currently underway as a single-dose challenge in men with combat-related PTSD for its potential in reducing the emotional strength of the PTSD-related traumatic memory (Clinical trials.gov).

\section{Electro-physiological approaches}

Considerable progress has been made in identifying neural circuits underlying fear learning, with prominent brain areas being the amygdala, hippocampus, ventromedial pre-frontal cortex (vmPFC), dorsal anterior cingulate cortex (dACC), and the insular cortex [60-65]. Newer imaging techniques show evidence of dysregulation of these neural circuits in PTSD [66, 67]. Specifically, functional imaging studies demonstrate that individuals with PTSD exhibit hyper-responsive amygdala activity to trauma or fear-related stimuli as well as during the resting state $[26,67-70]$. The impaired ability for extinction has been linked to decreased activity in the vmPFC and hippocampus, with hyperactivity demonstrated in the amygdala and dACC, all potential targets of neuromodulation by various means, including neural stimulation techniques used either alone or as augmentation to CBT $[71,72]$. While these methods are applied across neural networks, they likely involve reconsolidation at the cellular level, thus have been suggested as possible superior treatments for individuals with PTSD co-occurring with mild traumatic brain injury, in 
contrast to verbal treatments such as PE, since reconsolidation presupposes that the memory trace is persistently altered, not inhibited, and therefore is not dependent upon an intact vmPFC for modulation of amygdalar activity [73•].

Repetitive transcranial magnetic stimulation

In repetitive transcranial magnetic stimulation (rTMS), a magnetic coil is placed on the scalp, applying a powerful and variable magnetic field to the underlying neural tissues. In vitro and in vivo studies suggest that is allows fields of neurons to be depolarized or hyperpolarized depending on the frequency of stimulation (see Fitzgerald et al. for review) [74]. Current technology limits the penetration of the magnetic field in rTMS to just a few centimeters; thus, at this time, deep structures (e.g., the amygdala) are not viable targets for rTMS. Protocols that use rTMS to treat PTSD have thus largely been similar to existing FDA-approved protocols for MDD, which target the dorsolateral pre-frontal cortex (dlPFC), reported to be abnormal in PTSD [26]. To date, there have been eight published studies (132 total subjects); five of these studies were randomized, controlled trials, all recently reviewed by Karsen et al. [75••]. All targeted pre-frontal areas and most used a figure 8 coil, although other types of coils were used including angular, circular, and $\mathrm{H}$-coil. The main variables studied were effectiveness of treatment based on laterality (right vs left) and stimulation frequency $(0.3,1,5$, 10 , and $20 \mathrm{~Hz}$ ). In a double-blind placebo-controlled trial (30 subjects), Boggio et al. used high-frequency $(20 \mathrm{~Hz})$ transcranial magnetic stimulation (TMS) over ten sessions to compare the effectiveness of right versus left pre-frontal cortex stimulation for PTSD [76]. Both right- and left-sided treatment were effective in reducing PTSD symptoms compared to sham, with right-sided treatment (48.6\%) showing superior improvement over left-sided treatment $(22.8 \%)$ at post-treatment follow-up [76]. Treatment effects remained over months. Three of the randomized trials used rTMS alone. Two others tested TMS in combination with imaginal exposure. Osuch et al. used low-frequency ( $1 \mathrm{hz}$ ) inhibition over the right pre-frontal cortex and did not show benefit of TMS augmentation over exposure, whereas a trial by Isserles et al. which used high-frequency $(20 \mathrm{~Hz})$ stimulation of the medial pre-frontal cortex in combination with imaginal exposure showed benefit of this protocol over either TMS or exposure alone $[77,78]$. Treatment was generally well tolerated with reports of mild adverse effects of headaches and dizziness, and no major adverse effects reported $[75 \bullet \bullet]$.

Problematic for the theory underlying the use of high vs low frequency simulation is that both high (presumably stimulatory) and low (presumably inhibitory) frequency stimulation have both been shown to have benefits for PTSD symptoms. At this point, it is not clear if one protocol will become dominant as a method for using rTMS to treat PTSD or if several different protocols will be advanced. As technology improves, it is also possible that entirely different anatomical targets will be advanced.

Surgically implanted vagus nerve stimulation (sVNS) devices have been shown to be effective (now FDA approved) in the treatment of epilepsy and refractory depression $[79,80]$. The surgically implanted electrode is placed around the vagus nerve at the level of the neck, and does not require any craniotomy or 
direct access to the brain. The vagus nerve at the level of the neck carries efferent parasympathetic fibers (thought to comprise $20 \%$ of total vagus nerve fibers) as well as afferent sensory fibers (thought to comprise $80 \%$ of total vagus nerve fibers). With vagus nerve stimulation, there is activation of the nucleus solitary tract (NTS), then relaying of information to other targets that include the amygdala and hypothalamus [81]. Pre-clinical data demonstrate that amygdalar activation potentiates hippocampal activity, substantially enhancing memory consolidation. Clinical studies demonstrate enhanced neurocognitive performance with sVNS, perhaps a result of sVNS activation of polysynaptic norepinephrine projections to hippocampus, locus coeruleus and amygdala, as suggested by pre-clinical data $[82,83]$. Adding to this growing literature are recent pre-clinical findings that sVNS-potentiated fear extinction learning is correlated with facilitated long-term potentiation in the hippocampus [84, 85]. Thus, taken together, pre-clinical and emerging clinical evidences support the use of vagus nerve stimulation as a potential treatment for PTSD, either alone or as a combined physiological-psychotherapy augmentation approach. Currently, surgically implanted vagus nerve stimulation (VNS) is the only treatment available for PTSD; however, non-invasive VNS technology is being developed. There are no published treatment trials for PTSD as yet using these non-invasive devices.

Deep brain stimulation (DBS) is a surgically implantable technique in which an implanted electrode repeatedly administers electrical stimulation [86]. Electrode placement may vary, but the current targets remain subcortical structures [87•]. Initially, DBS had been used to effectively treat movement disorders including Parkinson's disease or other dystonias [88]. Currently, it is being investigated for the treatment of major depression disorder (MDD) and obsessive compulsive disorder (OCD). DBS for MDD treatment targets a number of brain areas: the subgenual cortex, ventral capsule/ventral striatum, and the nucleus accumbens, and is reported to be a success in $40-60 \%$ of recipients at 6 months to 1 year [89-92]. In addition to MDD, OCD has been successfully treated with DBS $[93,94]$. Similar success has been noted in pre-clinical DBS studies of the basolateral nucleus of the amygdala (a key node in the fear learning network) that resulted in a decrease in fear-related behavior [95, 96]. A single clinical trial is currently ongoing of DBS to the basolateral nucleus of the amygdala to treat PTSD [97].

\section{Other physiological technologies}

Other potential technologies are 1) direct current stimulation (tDCS), a noninvasive neuromodulation technique using low current electrodes placed on the surface of the scalp that deliver current though the skull to modify cortical excitability; 2) cranial electrical stimulation (CES), a low-level alternating electrical current; 3 ) transcranial alternating current stimulation (tACS) which is similar to CES in that it uses a low-intensity alternating current applied to the head; and 4) electroconvulsive therapy (ECT) which is established as an effective therapy for treatment-resistant depression. While some of these methods show effects on memory and fear learning, none, as yet, has been studied for efficacy in treating PTSD except for ECT. In a single prospective study, six 


\section{Conclusion}

bilateral ECT treatments were carried out twice weekly in 20 patients with medication refractory PTSD, resulting in $40 \%$ decrease in the ClinicianAdministered Post-traumatic Stress Disorder Scale scores with a total response rate of $82 \%$ [98]. This treatment gain was sustained up to 4 years later.

Overall, clinical care recommendations over the past decade have continued to rely on SSRI, prazosin, as first-line treatment for nightmares. During this time, however, there have been significant advancements in understanding the neurobiology of fear learning and extinction, and the role of specific neural circuits in this learning. These concepts are now at the center of a renaissance in PTSD treatment research and development.

\section{Compliance with Ethics Guidelines}

\section{Conflict of Interest}

Dewleen G Baker declares that she has no conflict of interest, Immanuel Lerman declares that he has no conflict of interest, Emmanuel P. Espejo declares that he has no conflict of interest, and Robert McLay declare declares that he has no conflict of interest.

\section{Human and Animal Rights and Informed Consent}

This article does not contain any studies with human or animal subjects performed by any of the authors.

\section{References and Recommended Reading}

Papers of particular interest, published recently, have been highlighted as:

- Of importance

- Of major importance

1. Diagnostic and Statistical Manual of Mental Disorders (5th

6. Green BL, Grace MC, Lindy JD, et al. Risk factors for ed., DSM-V); American Psychiatric Association, Washington, D.C. 2013.

2. Breslau N, Davis GC, Andreski P, Peterson E. Traumatic events and posttraumatic stress disorder in an urban population of young adults. Arch Gen Psychiatry. 1991;48(3):216-22.

3. Frans O, Rimmo PA, Aberg L, Fredrikson M. Trauma exposure and post-traumatic stress disorder in the general population. Acta Psychiatr Scand. 2005;111(4):291-9.

4. Darves-Bornoz JM, Alonso J, de Girolamo G, et al. Main traumatic events in Europe: PTSD in the European study of the epidemiology of mental disorders survey. J Trauma Stress. 2008;21(5):455-62.

5. Kessler RC, Sonnega A, Bromet E, et al. Posttraumatic stress disorder in the national comorbidity survey. Arch Gen Psychiatry. 1995;52(12):1048-60. PTSD and other diagnoses in a general sample of Vietnam veterans. Am J Psychiatry. 1990;147(6):729-33.

7. Wolfe J, Chrestman KR, Ouimette PC, et al. Traumarelated psychophysiological reactivity in women exposed to war-zone stress. J Clin Psychol. 2000;56(10):1371-9.

8. Stein MB, Jang KL, Taylor S, et al. Genetic and environmental influences on trauma exposure and posttraumatic stress disorder symptoms: a twin study. Am J Psychiatry. 2002;159(10):1675-81.

9. $\quad$ Yurgil KA, Barkauskas DA, Vasterling JJ, Nievergelt CM, Larson GE, Schork NJ, et al. Association between traumatic brain injury and risk of posttraumatic stress disorder in active-duty Marines. JAMA Psychiatry. 2014;71(2):149-57.

This manuscript uses pre-and post-deployment data to provide prospective evidence for an association between traumatic brain injury and increased risk for PTSD. 
10. Mohamed S, Rosenheck RA. Pharmacotherapy of PTSD in the U.S. department of veterans affairs: diagnosticand symptom-guided drug selection. J Clin Psychiatry. 2008;69(6):959-65.

This manuscript which reviews data on the use of medications for treatment of PTSD, shows that diverse psychotropic medication classes are extensively used for the treatment of PTSD in the VA.

11. Harpaz-Rotem I, Rosenheck RA, Mohamed S, Desai RA. Pharmacologic treatment of posttraumatic stress disorder among privately insured Americans. Psychiatr Serv. 2008;59(10):1184-90.

$12 . \bullet$ Baker DG, Nievergelt CM, Risbrough VB. Post-traumatic stress disorder: emerging concepts of pharmacotherapy. Expert Opin Emerg Drugs. 2009;14(2):25172.

This manuscript provides a comprehensive review of pharmacologic PTSD treatment studies.

13. Hermes E, Rosenheck R. Prevalence, pharmacotherapy and clinical correlates of diagnosed insomnia among veterans health administration service users nationally. Sleep Med. 2014;15(5):508-14.

14. Hermes E, Harpaz-Rotem I, Rosenheck R. Diffusion of prazosin treatment for PTSD. Am J Psychiatry. 2014;171(1):117.

15. Institute of Medicine (IOM). Committee on Treatment of Posttraumatic Stress Disorder. Treatment of posttraumatic stress disorder: an assessment of the evidence. Washington: National Academies Press; 2008.

16. National Collaborating Centre for Mental Health, National Institute for Health and Clinical Excellence. Posttraumatic stress disorder: the management of PTSD in adults and children in primary and secondary care. London: published by Gaskell and the British Psychological Society, 2005

17. Stein DJ, Rothbaum BO, Baldwin DS, Szumski A, Pedersen R, Davidson JR. A factor analysis of posttraumatic stress disorder symptoms using data pooled from two venlafaxine extended-release clinical trials. Brain Behav. 2013;3(6):738-46.

18. Rothbaum BO, Davidson JR, Stein DJ, Pedersen R, Musgnung J, Tian XW, et al. A pooled analysis of gender and trauma-type effects on responsiveness to treatment of PTSD with venlafaxine extended release or placebo. J Clin Psychiatry. 2008;69(10):1529-39.

19. Green B. Prazosin in the treatment of PTSD. J Psychiatr Pract. 2014;20(4):253-9.

This manuscript provides a comprehensive review of PTSD prazosin treatment studies.

20. Steckler T, Risbrough V. Pharmacological treatment of PTSD_established and new approaches. Neuropharmacology. 2011;62(2):617-27.

21. Hauger RL, Olivares-Reyes JA, Dautzenberg FM, Lohr JB, Braun S, Oakley RH. Molecular and cell signaling targets for PTSD pathophysiology and pharmacotherapy. Neuropharmacology. 2012;62(2):705-14.

22. Nievergelt CM, Maihofer AX, Maja Mustapic M, Yurgil KA, Schork NJ, Miller MW, et al. Genomic predictors of combat stress vulnerability and resilience in U.S.
Marines: a multi-ethnic genome-wide association study implicates PRTFDC1 as a potential PTSD gene. Psychoneuroendocrinology. 2015;51:459-71.

23. Stefanovics EA, Krystal JH, Rosenheck RA. Symptom structure and severity: a comparison of responses to the positive and negative syndrome scale (PANSS) between patients with PTSD or schizophrenia. Compr Psychiatry. 2014;55(4):887-95.

24. Krystal JH, Rosenheck RA, Cramer JA, Vessicchio JC, Jones KM, Vertrees JE, et al. Adjunctive risperidone treatment for antidepressant-resistant symptoms of chronic military service-related PTSD: a randomized trial. JAMA. 2011;306(5):493-502.

25. Reul JM. Making memories of stressful events: a journey along epigenetic, gene transcription, and signaling pathways. Front Psychiatry. 2014;5:5.

26. Huang MX, Yurgil KA, Robb A, Angeles A, Diwakar M, Risbrough VB, et al. Voxel-wise resting-state MEG source magnitude imaging study reveals neurocircuitry abnormality in active-duty service members and veterans with PTSD. Neuroimage Clin. 2014;5:408-19.

27. Herry C, Johansen JP. Encoding of fear learning and memory in distributed neuronal circuits. Nat Neurosci. 2014;17(12):1644-54.

28. Rozeske RR, Valerio S, Chaudun F, Herry C. Prefrontal neuronal circuits of contextual fear conditioning. Genes Brain Behav. 2014 Oct 7. [Epub ahead of print]

29.• Nader K, Hardt O, Lanius R. Memory as a new therapeutic target. Dialogues Clin Neurosci. 2013;15(4):475-86. Review.

This manuscript reviews and discusses the benefits and possible pitfalls of focusing on memory as a therapeutic target.

30. Bisaz R, Travaglia A, Alberini CM. The neurobiological bases of memory formation: from physiological conditions to psychopathology. Psychopathology. 2014;47(6):347-56.

31. Rosenberg T, Gal-Ben-Ari S, Dieterich DC, Kreutz MR, Ziv NE, Gundelfinger ED, et al. The roles of protein expression in synaptic plasticity and memory consolidation. Front Mol Neurosci. 2014;7:86.

32. Kwapis JL, Wood MA. Epigenetic mechanisms in fear conditioning: implications for treating post-traumatic stress disorder. Trends Neurosci. 2014 Sep 11. [Epub ahead of print]

33. Forcato C, Fernandez RS, Pedreira ME. Strengthening a consolidated memory: the key role of the reconsolidation process. J Physiol Paris. 2014 Sep 16. [Epub ahead of print]

34. Itzhak Y, Perez-Lanza D, Liddie S. The strength of aversive and appetitive associations and maladaptive behaviors. IUBMB Life. 2014;66(8):559-71.

35. Quirk GJ, Mueller D. Neural mechanisms of extinction learning and retrieval. Neuropsychopharmacology. 2008;33(1):56-72.

36. Piñeyro ME, Ferrer Monti RI, Alfei JM, Bueno AM, Urcelay GP. Memory destabilization is critical for the success of the reactivation-extinction procedure. Learn Mem. 2013; 21(1):46-54. Erratum in: Learn Mem. 2014 Feb;21(2):127 
37.• Singewald N, Schmuckermair C, Whittle N, Holmes A, Ressler KJ. Pharmacology of cognitive enhancers for exposure-based therapy of fear, anxiety and traumarelated disorders. Pharmacol Ther. 2014 Dec 27. (14)00233-2. doi: 10.1016/j.pharmthera.2014.12.004. This manuscript provides a review of pharmacologic compounds that show evidence of being fear extinction enhancers.

38. Otis JM, Werner CT, Mueller D. Noradrenergic regulation of fear and drug-associated memory reconsolidation. Neuropsychopharmacology. 2014 Sep 12. [Epub ahead of print]

39. Lonergan MH, Olivera-Figueroa LA, Pitman RK, Brunet A. Propranolol's effects on the consolidation and reconsolidation of long-term emotional memory in healthy participants: a meta-analysis. J Psychiatry Neurosci. 2013;38(4):222-31.

40.• Agren T. Human reconsolidation: a reactivation and update. Brain Res Bull. 2014;105:70-82.

This manuscript defines consolidation and reviews the literature on pharmaceutical compounds thought to affect reconsolidation.

41. Foa EB. Prolonged exposure therapy: past, present, and future. Depress Anxiety. 2011;28(12):1043-7.

42. Resick PA, Nishith P, Weaver TL, Astin MC, Feuer CA. A comparison of cognitive-processing therapy with prolonged exposure and a waiting condition for the treatment of chronic posttraumatic stress disorder in female rape victims. J Consult Clin Psychol. 2002;70(4):867-79.

43. Resick PA, Galovski TE, Uhlmansiek MO, Scher CD, Clum GA, Young-Xu Y. A randomized clinical trial to dismantle components of cognitive processing therapy for posttraumatic stress disorder in female victims of interpersonal violence. J Consult Clin Psychol. 2008;76(2):243-58.

44. Merlo E, Milton AL, Goozée ZY, Theobald DE, Everitt BJ. Reconsolidation and extinction are dissociable and mutually exclusive processes: behavioral and molecular evidence. J Neurosci. 2014;34(7):2422-31.

45. Flavell CR, Lambert EA, Winters BD, Bredy TW. Mechanisms governing the reactivation-dependent destabilization of memories and their role in extinction. Front Behav Neurosci. 2013;7:214.

46. Hofmann SG. Cognitive processes during fear acquisition and extinction in animals and humans: implications for exposure therapy of anxiety disorders. Clin Psychol Rev. 2008;28(2):199-210.

47. Kar N. Cognitive behavioral therapy for the treatment of post-traumatic stress disorder: a review. Neuropsychiatr Dis Treat. 2011;7:167-81.

48. Bauer EP. Serotonin in fear conditioning processes. Behav Brain Res. 2015;277C:68-77.

49. Passamonti L, Crockett MJ, Apergis-Schoute AM, Clark L, Rowe JB, Calder AJ, et al. Effects of acute tryptophan depletion on prefrontal-amygdala connectivity while viewing facial signals of aggression. Biol Psychiatry. 2012;71(1):36-43.
50. Schneier FR, Neria Y, Pavlicova M, Hembree E, Suh EJ, Amsel L, et al. Combined prolonged exposure therapy and paroxetine for PTSD related to the World Trade Center attack: a randomized controlled trial. Am J Psychiatry. 2012;169:80-8.

51. de Kleine RA, Rothbaum BO, van Minnen A. Pharmacological enhancement of exposure-based treatment in PTSD: a qualitative review. Eur J Psychotraumatol. 2013 Oct 17; 4.

This manuscript gives a review of studies of the pharmacological enhancement of exposure therapy in PTSD.

52. Walker DL, Ressler KJ, Lu KT, Davis M. Facilitation of conditioned fear extinction by systemic administration or intra-amygdala infusions of D-cycloserine as assessed with fear-potentiated startle in rats. J Neurosci. 2002;22:2343-51.

53. Hofmann SG, Otto MW, Pollack MH, Smits JA. Dcycloserine augmentation of cognitive behavioral therapy for anxiety disorders: an update. Curr Psychiatry Rep. 2015;17(1):532.

54. Litz BT, Salters-Pedneault K, Steenkamp MM, Hermos JA, Bryant RA, Otto MW, et al. A randomized placebocontrolled trial of D-cycloserine and exposure therapy for posttraumatic stress disorder. J Psychiatr Res. 2012;46(9):1184-90.

55. de Kleine RA, Hendriks GJ, Kusters WJ, Broekman TG, van Minnen A. A randomized placebo-controlled trial of D-cycloserine to enhance exposure therapy for posttraumatic stress disorder. Biol Psychiatry. 2012;71(11):962-8.

56. Difede J, Cukor J, Wyka K, Olden M, Hoffman H, Lee FS, et al. D-Cycloserine augmentation of exposure therapy for post-traumatic stress disorder: a pilot randomized clinical trial. Neuropsychopharmacology. 2014;39(5):1052-8.

57. Rothbaum BO, Price $M$, Jovanovic T, Norrholm SD, Gerardi M, Dunlop B, et al. A randomized, doubleblind evaluation of D-cycloserine or alprazolam combined with virtual reality exposure therapy for posttraumatic stress disorder in Iraq and Afghanistan War veterans. Am J Psychiatry. 2014;171(6):640-8.

58.• Hofmann SG. D-cycloserine for treating anxiety disorders: making good exposures better and bad exposures worse. Depress Anxiety. 2014;31(3):175-7.

This manuscript defines conceptual overview of the benefits and risks of use of the cognitive enhancer, $\mathrm{d}$-cycloserine, presaging likely similar issues with human use of other similar putative cognitive enhancers.

59. Bolkan SS, Lattal KM. Opposing effects of Dcycloserine on fear despite a common extinction duration: interactions between brain regions and behavior. Neurobiol Learn Mem. 2014;113:25-34.

60. Milad MR, Quirk GJ. Fear extinction as a model for translational neuroscience: ten years of progress. Annu Rev Psychol. 2012;63:129-51.

61. Pare D, Duvarci S. Amygdala microcircuits mediating fear expression and extinction. Curr Opin Neurobiol. 2012;22(4):717-23. 
62. Martin EI, Ressler KJ, Binder E, Nemeroff CB. The neuro- biology of anxiety disorders: brain imaging, genetics, and psychoneuroendocrinology. Psychiatr Clin N Am. 2009;32(3):549-75.

63. LeDoux J. The emotional brain, fear, and the amygdala. Cell Mol Neurobiol. 2003;23(4-5):727-38.

64. Hartley CA, Phelps EA. Changing fear: the neurocircuitry of emotion regulation. Neuropsychopharmacol Off Publ Am Coll Neuropsychopharmacol. 2010;35:136-46.

65. Rosen GM, Lilienfeld SO. Posttraumatic stress disorder: an empirical evaluation of core assumptions. Clin Psychol Rev. 2008;28:837-68.

66. Rauch SL, Shin LM, Phelps EA. Neurocircuitry models of posttraumatic stress disorder and extinction: human neuroimaging research-past, present, and future. Biol Psychiatry. 2006;60:376-82.

67. Rauch SL, Shin LM, Whalen PJ, Pitman RK. Neuroimaging and the neuroanatomy of PTSD. CNS Spectr. 1998;3 Suppl 2:30-41.

68. Shin LM, Orr SP, Carson MA, Rauch SL, Macklin ML, Lasko NB, et al. Regional cerebral blood flow in the amygdala and medial prefrontal cortex during traumatic imagery in male and female Vietnam veterans with PTSD. Arch Gen Psychiatry. 2004;61:168-76.

69. Chung YA, Kim SH, Chung SK, Chae JH, Yang DW, Sohn HS, et al. Alterations in cerebral perfusion in posttraumatic stress disorder patients without reexposure to accident-related stimuli. Clin Neurophysiol Off J Int Fed Clin Neurophysiol. 2006;117:637-42.

70. Semple WE, Goyer PF, McCormick R, Donovan B, Muzic Jr RF, Rugle L, et al. Higher brain blood flow at amygdala and lower frontal cortex blood flow in PTSD patients with comorbid cocaine and alcohol abuse compared with normals. Psychiatry. 2000;63:65-74.

71. Milad MR, Quirk GJ, Pitman RK, Orr SP, Fischl B, Rauch SL. A role for the human dorsal anterior cingulate cortex in fear expression. Biol Psychiatry. 2007;62(10):1191-4.

72. Milad MR, Rosenbaum BL, Simon NM. Neuroscience of fear extinction: implications for assessment and treatment of fear-based and anxiety related disorders. Behav Res Ther. 2014 Aug 23. [Epub ahead of print]

73.• Schiller D, Kanen JW, LeDoux JE, Monfils MH, Phelps EA. Extinction during reconsolidation of threat memory diminishes prefrontal cortex involvement. Proc Natl Acad Sci U S A. 2013;110(50):20040-5.

Results of this pre-clinical study suggest that extinction learning during reconsolidation prevents the return of defensive reactions and diminishes prefrontal cortical involvement. This is important because reducing the necessity for the prefrontalcortical circuit to control defensive behaviors may help overcome an impediment in the long term efficacy of current PTSD treatments.

74. Fitzgerald PB, Fountain S, Daskalakis ZJ. A comprehensive review of the effects of rTMS on motor cortical excitability and inhibition. Clin Neurophysiol. 2006;117(2):2584-96.
75.• Karsen EF, Watts BV, Holtzheimer PE. Review of the effectiveness of transcranial magnetic stimulation for post-traumatic stress disorder. Brain Stimul. 2014;7(2):151-7.

This manuscript gives a review of use of transcranial magnetic stimulation in PTSD.

76. Boggio PS, Rocha M, Oliveira MO, et al. Noninvasive brain stimulation with high-frequency and lowintensity repetitive transcranial magnetic stimulation treatment for posttraumatic stress disorder. J Clin Psychiatry. 2010;71(8):992e9.

77. Osuch EA, Benson BE, Luckenbaugh DA, Geraci M, Post RM, McCann U. Repetitive TMS combined with exposure therapy for PTSD: a preliminary study. J Anxiety Disord. 2009;23(1):54e9.

78. Isserles $\mathrm{M}$, Rosenberg $\mathrm{O}$, Dannon P, Levkovitz Y, Kotler $\mathrm{M}$, Deutsch $\mathrm{F}$, et al. Cognitive-emotional reactivation during deep transcranial magnetic stimulation over the prefrontal cortex of depressive patients affects antidepressant outcome. J Affect Disord. 2011;128(3):235-42.

79. Handforth A, DeGiorgio CM, Schachter SC. Vagus nerve stimulation therapy for partial onset seizures: a randomized active-control trial. Neurology. 1998; $51: 48$

80. Nierenberg AA, Alpert JE, Gardner-Schuster EE. Vagus nerve stimulation: 2-year outcomes for bipolar versus unipolar treatment-resistant depression. Biol Psychiatry. 2008;64:455-60.

81. George MS, Sackeim HA, Rush AJ, et al. Vagus nerve stimulation: a new tool for brain research and therapy. Biol Psychiatry. 2000;47(4):287-95.

82. Clark KB, Naritoku DK, Smith DC, Browning RA, Jensen RA. Enhanced recognition memory following vagus nerve stimulation in human subjects. Nat Neurosci. 1999;2(1):94-8.

83. Hassert DL, Miyashita T, Williams CL. The effects of peripheral vagal nerve stimulation at a memorymodulating intensity on norepinephrine output in the basolateral amygdala. Behav Neurosci. 2004;118(1):79-88.

84. Peña DF, Engineer ND, McIntyre CK. Rapid remission of conditioned fear expression with extinction training paired with vagus nerve stimulation. Biol Psychiatry. 2013;73(11):1071-7.

85. Peña DF, Childs JE, Willett S, Vital A, McIntyre CK, Kroener S. Vagus nerve stimulation enhances extinction of conditioned fear and modulates plasticity in the pathway from the ventromedial prefrontal cortex to the amygdala. Front Behav Neurosci. 2014;8(September):327.

86. Rowny, S. B., \& Lisanby, S. H. (2008). Brain stimulation in psychiatry. In A. Tasman, J. Kay, J.A. Lieberman, M. B. First, \& M. Maj (Eds.), Psychiatry (3rd ed., pp. 2354-2371). New York: Wiley

87. Rosa M, Giannicola G, Marceglia S, Fumagalli M, Barbieri S, Pri-ori A. Neurophysiology of deep brain stimulation. Int Rev Neurobiol. 2012;107:23-55.

This manuscript provides a review of the data concerning the neurophysiology of deep brain stimulation in humans. 
88. Yu H, Neimat JS. The treatment of movement disorders by deep brain stimulation. Neurother J Am Soc Exp Neurother. 2008;5(1):26-36.

89. Mayberg H. Targeted electrode-based modulation of neural circuits for depression. J Clin Investig. 2009;119:717-25.

90. Puigdemont D, Pérez-Egea R, Portella MJ, et al. Deep brain stimulation of the subcallosal cingulate gyrus: further evidence in treatment-resistant major depression. Int J Neuropsychopharmacol. 2011;15:1-13.

91. Malone DA, Dougherty DD, Rezai AR, Carpenter LL, et al. Deep brain stimulation of the ventral capsule/ ventral striatum for treatment-resistant depression. Biol Psychiatry. 2009;65:267-75.

92. Bewernick BH, Hurlemann R, Matusch A, Kayser S, et al. Nucleus accumbens deep brain stimulation decreases ratings of depression and anxiety in treatmentresistant depression. Biol Psychiatry. 2010;67:110-6.

93. Goodman WK, Alterman RL. Deep brain stimulation for intractable psychiatric disorders. Annu Rev Med. 2012;63:511-24.
94. Denys D, Mantione $\mathrm{M}$, Figee $\mathrm{M}$, et al. Deep brain stimulation of the nucleus accumbens for treatmentrefractory obsessive-compulsive disorder. Arch Gen Psychiatry. 2010;67(10):1061-8.

95. Langevin J-P, De Salles AAF, Kosoyan H, Krahl S. Deep brain stimulation alleviates posttraumatic stress disorder in a rat model. J Psychiatr Res. 2010;44:1241-2145.

96. Stidd DA, Vogelsang K, Krahl SE, Langevin JP, Fellous JM. Amygdala deep brain stimulation is superior to paroxetine treatment in a rat model of posttraumatic stress disorder. Brain Stimul. 2013;6:837-44.

97. Koek RJ, Langevin J-P, Krahl SE, et al. Deep brain stimulation of the basolateral amygdala for treatmentrefractory combat post-traumatic stress disorder (PTSD): study protocol for a pilot randomized controlled trial with blinded, staggered onset of stimulation. Trials. 2014;15:356. doi:10.1186/1745-6215-15-356.

98. Margoob MA, Ali Z, Andrade C. Efficacy of ECT in chronic, severe, antidepressant- and CBT-refractory PTSD: an open, prospective study. Brain Stimulation. 2010;3(1):28-35. 